\title{
Bilateral Control of Hindlimb Scratching in the Spinal Turtle: Contralateral Spinal Circuitry Contributes to the Normal Ipsilateral Motor Pattern of Fictive Rostral Scratching
}

\author{
Paul S. G. Stein,, ${ }^{1,2}$ John C. Victor, ${ }^{1}$ Edelle C. Field, ${ }^{1,2}$ and Scott N. Currie ${ }^{3}$ \\ 'Department of Biology and ${ }^{2}$ Movement Science Program, Washington University, St. Louis, Missouri 63130 and \\ ${ }^{3}$ Department of Neuroscience, University of California at Riverside, Riverside, California 92521
}

In a spinal turtle, unilateral stimulation in the rostral scratch receptive field elicited rhythmic fictive rostral scratching in ipsilateral hindlimb motor neurons; contralateral hip motor activity was also rhythmic and out-ofphase with ipsilateral hip motor activity. When left and right rostral scratch receptive fields were stimulated simultaneously, bilateral rhythmic fictive rostral scratching was produced; left hindlimb scratching was out-of-phase with right hindlimb scratching. Thus, spinal circuits coordinate interlimb phase during bilateral fictive scratching.

We examined the contributions of contralateral spinal circuitry to the normal pattern of right hindlimb fictive rostral scratching by removing the left halves of the $D 7 \mathrm{seg}$ ment and the hindlimb enlargement (D8-S2 segments). After left-hemicord removal, stimulation in the right rostral scratch receptive field usually elicited a variation of rostral scratching with rhythmic right hip flexor activity and no right hip extensor activity; thus, right hip flexor rhythm generation does not require left hindlimb enlargement circuitry. Normal right hindlimb rostral scratching with rhythmic alternation between hip flexor and extensor activities was rarely observed; thus, contralateral spinal circuitry contributes to the production of normal ipsilateral fictive rostral scratching. After left-hemicord removal, stimulation in the left rostral scratch receptive field elicited rhythmic right hip extensor activity; thus, contralateral spinal circuitry can generate a hip extensor rhythm during ipsilateral rostral scratch receptive field stimulation.

Our observations and those of Berkowitz and Stein (1994a,b) support the concept that an ipsilateral hindlimb's normal rostral scratch motor pattern is generated by a modular central pattern generator that is bilaterally distributed in the spinal cord.

[Key words: spinal, scratching, tactile, fictive, turtle, central pattern generator]

The turtle spinal cord contains central pattern generators (CPGs) for ipsilateral hindlimb rostral scratching (Rohertson et al., 1985). For example, the right rostral scratch CPG is the set of

\footnotetext{
Received Oct. 3, 1994; revised Jan. 4, 1994; accepted Jan. 9, 1994.

We thank Dr. Ari Berkowitz for editorial assistance, Dr. Gavin Perry for software development, and Angie Rosengarten for her participation in several of the bilateral-recording experiments. This work was supported by NIH Grants NS15049 and NS30786 to P.S.G.S. and NSF Grant IBN93-08804 to S.N.C.

Correspondence should he addressed to Dr. Paul S. G. Stein, Department of Biology, Washington University, St. Louis, MO 63130

Copyright (C) 1995 Society for Neuroscience $0270-6474 / 95 / 154343-13 \$ 05.00 / 0$
}

spinal cord neurons that can generate the normal motor pattern of right hindlimb rostral scratching without movement-related sensory feedback (Stein, 1989). Right hindlimb rostral scratching is elicited by tactile stimulation in the right rostral scratch receptive field. A spinal turtle with neuromuscular blockade produces a normal electroneurographic motor pattern (ENG) of fictive rostral scratching that is an excellent replica of the normal electromyographic motor pattern (EMG) recorded during rostral scratching movements (Robertson et al., 1985).

Most previous work with turtle scratching has focused upon neural activity ipsilateral to the stimulation site (Robertson et al., 1985; Stein, 1989). The normal fictive motor pattern of right rostral scratching includes rhythmic alternation between right hip flexor (VP-HP) and right hip extensor (HR-KF) activity; see Matcrials and Methods for full names of ncrves. The right hip flexor is quiescent when the right hip extensor is active. Right knee extensors (FT-KE and AM-KE) are active during the latter portion of right hip flexor activity. Implied, but not directly stated in this earlier work, is the hypothesis that the ipsilateral rostral scratch CPG is located entirely within the ipsilateral spinal cord.

Contralateral motor neurons are active during scratching by an ipsilateral limb, however (Deliagina et al., 1981; O'Donovan et al., 1982; Currie and Stein, 1989; Berkowitz and Stein, 1994a,b). This article examines contralateral motor activity during fictive rostral scratching. Contralateral interneurons that excite these motor neurons are most likely also active. Experiments with fos expression (Barajon et al., 1992) and unit recordings from spinal interneurons (Arshavsky et al., 1978; Berkowitz and Stein, 1994a) provide evidence of bilateral activation of spinal interneurons during ipsilateral scratching in cat and turtle. Some of these interneurons may be members of a scratch CPG; thus, these data support the hypothesis that a scratch CPG for a single limb includes neural elements on both sides of the spinal cord. This paper presents additional evidence that supports this hypothesis for turtle rostral scratching.

The "hip-extensor deletion" is a spontaneous variation of normal fictive rostral scratching in the turtle. During a rostral scratch cycle with a hip-extensor deletion, there is no hip flexor quiescence and no hip extensor activation (Stein et al., 1982; Robertson and Stein, 1988). Instead, a hip flexor burst immediately follows the previous hip flexor burst; each of these hip flexor bursts displays the characteristic intensity modulations of hip flexor bursts generated during normal rostral scratching. During the latter portion of each hip flexor burst, there is activation of the knee extensors FT-KE and AM-KE. After bilateral 
removal of posterior segments of the hindlimb enlargement, Mortin and Stein (1989) demonstrated that rostral scratching responses mainly involved cycles with hip-extensor deletions.

In this article, we examine the contributions of left spinal circuitry to the production of the normal pattern of rostral scratching, with rhythmic alternation between right hip flexor and right hip extensor bursts. After unilateral removal of the left hindlimb enlargement, stimulation in the right rostral scratch receptive field elicits mainly right hip-extensor deletions, a variation of fictive rostral scratching. Our results support the hypothesis that contralateral circuitry plays an important role in the production of the normal ipsilateral rostral scratching motor pattern.

The present results have been presented previously in abstract form (Stein et al., 1994).

\section{Materials and Methods}

Surgical preparation. Red-eared turtles $(n=18)$, Trachemys scripta elegans (formerly Pseudemys scripta elegans), 330-750 gm, were placed on crushed ice for at least 1 hr prior to surgery to induce hypothermic analgesia (Melby and Altman, 1974). Every turtle was then spinalized just caudal to the forelimb enlargement by complete spinal transection midway between the $\mathrm{D}^{2} 2$ (dorsal 2 , second post-cervical spinal segment) and the D3 dorsal roots (see Figs. 1, 6A). After spinal transection, the cord was covered with Gelfoam surgical sponges (Upjohn, Kalamazoo, MI) soaked in turtle saline (Robertson et al., 1985) and the opening was sealed with a wax plug that was glued to the shell.

We used two types of experimental preparations. In the first type, termed "bilateral-recording preparation" $(n=11)$, we recorded bilaterally from hindlimb motor nerves during unilateral and bilateral stimulation in the rostral scratch receptive fields (see Fig. 1). In the second type, termed "left-hemicord removal preparation" $(n=7)$, we recorded from right hindlimb motor nerves during unilateral stimulation in either the right rostral scratch receptive field or the left rostral scratch receplive field (see Fig. 6A). After control recordings were obtained in the second type of preparation, we removed the left halves of six spinal-cord segments, D7-D10, S1, and S2. The D8, D9, and D10 spinal cord segments are just anterior to the two sacral spinal segments, S1 and S2. Somata of hip flexors and knee extensors reside in D8 and D9; somata of hip extensors reside in D9, D10, S1, and S2 (Ruigrok and Crowe, 1984). Segments D8-S2 are collectively termed the hindlimb enlargement. Mortin and Stein (1989) demonstrated that D7-D10 contain circuitry critical for the production of normal rostral scratching motor patterns. After left-hemicord removal, we then repeated the stimulation paradigms that were used before the left-hemicord removal. All surgical procedures were performed while the turtle was maintained in crushed ice with the exception of the left-hemicord removal; this procedure was performed at room temperature after obtaining control recordings.

In the bilateral-recording preparation, the only spinal cord exposure was that required for transection of the D2-D3 segments. In the lefthemicord removal preparation, the D7-D $10, \mathrm{~S} 1$, and $\mathrm{S} 2$ segments were also exposed and bathed in turtle saline. In both types of preparation, hip flexor (HF), hip extensor (HE), and knee extensor (KE) hindlimb muscle nerves on the right side were dissected free for $\mathrm{ENG}$ recordings (Robertson et al., 1985; Mortin and Stein, 1989). VP-HP, the hip flexor (protractor) muscle nerve innervating puboischiofemoralis internus, pars anteroventralis, was dissected in all preparations. At least one of the following knee extensor nerves was dissected: AM-KE (triceps femoris, pars ambiens) or FT-KE (triceps femoris, pars femorotibialis). In addition, in some of the bilateral-recording preparations $(n=9)$ and in all of the left-hemicord removal preparations $(n=7)$, the HR-KF nerve, innervating flexor cruris, pars flexor tibialis internus and several other muscles that extend (retract) the hip and flex the knee, was dissected. In all bilateral-recording preparations, the corresponding nerves were also dissected on the left side.

After completion of these surgical procedures, each turtle was removed from the ice, allowed to warm to room temperature, and immobilized with gallamine (Flaxedil; American Cyanamid, Pearl River, $\mathrm{NY}$ ), a neuromuscular blocking agent, at a dosage of $8 \mathrm{mg} / \mathrm{kg}$ body weight. Dental wax (Modern Materials Red Utility Wax Strips, Miles, South Bend, IN) was molded into wells to surround each of the surgically exposed regions; the wells were glued to the shell with Permabond
910 adhesive (Permabond, Englewood, NJ). Each peripheral-nerve well was filled with mineral oil. The trachea was intubated and the turtle maintained on artificial respiration for the remainder of the experiment.

In the left-hemicord removal preparation, after completion of control recordings during stimulation in the right and the left rostral scratch receptive fields, the left halves of six spinal segments (D7-D10, S1, S2) were completely removed. The initial step of left-segment removal was removal of the dorsal portions of these segments' meninges. We then separated the left spinal cord from the right spinal cord in segments D7-S2 with a longitudinal midline section. We used the prominent stripe that runs along the dorsal midline of the spinal cord at the junction of the left and the right dorsal funiculi to assist our placement of the microscalpel for the midline section. The microscalpel was gently moved in an anterior/posterior motion to cut down the midline between the left and right dorsal funiculi and through the spinal gray and white commissures. The next cut was a left hemisection at the border of the D6 and D7 segments. Then, the left hemicord in segment D7 was held with forceps and gently pulled back; as the left hemicord was pulled back in segments D7-S2, any remaining connection of the left hemicord with the right hemicord was completely severed with fine iridectomy scissors. All dorsal and ventral roots on the left side of segments D7S2 were completely cut. Following complete left/right separation of the S2 segment, the left half of the spinal cord was transected at the border of the S2 segment and the Cal (Caudal 1) segment; this completely isolated the left halves of segments D7-D10, S1 and S2 from the remainder of the spinal cord. The isolated left hemicord was then completely removed. Physiological recordings were obtained after a recovery period of $1-2 \mathrm{hr}$.

Recordings. ENGs from each nerve were obtained using a pair of $100 \mu \mathrm{m}$ diameter silver wire electrodes immersed in the mineral oil pool. The ENGs were amplified ( $100-1000 \mathrm{~Hz}$ bandpass) and stored on DAT tape (DC-5 kHz bandpass) for later analyses and hard-copy printouts.

Stimulation. Rostral scratch motor patterns were elicited by stimulation of specific sites in the right and the left rostral scratch receptive fields (Stein and Grossman, 1980; Mortin et al., 1985; Mortin and Stein, 1990). In most preparations, we stimulated SP2, the most anterior site on the inguinal plate of the shell bridge (Mortin and Stein, 1990). We used mechanical stimulation applied with the smooth, fire-polished end of a glass rod; in experiments with only unilateral stimulation, we attached the glass rod to a hand-held force transducer. We elicited fictive rostral scratching in some bilateral-recording preparations with the following mechanical stimulation paradigm: stimulate on one side for several seconds, continue that stimulation and also stimulate on the other side for several seconds, discontinue stimulation on the first side, and continue stimulation only on the other side for several more seconds. In other bilateral-recording preparations, we elicited fictive rostral scratching by delivering electrical pulses to each side via two closely spaced pins placed in each SP2 (Currie and Stein, 1990). We usually delivered trains of 33 pulses with $320 \mathrm{msec}$ interpulse interval; each pulse was $2-10 \mathrm{~V}$ with a duration of $1 \mathrm{msec}$. In these bilateral-recording preparations, we delivered the trains of electrical stimulation in blocks of six according to the following sequence: left, right, bilateral, bilateral, left, right. We allowed 2-3 min recovery between each stimulus presentation. During bilateral stimulation, pulses were delivered simultaneously to the left and right sides.

Spinal cord morphology in the left-hemicord removal preparation. After all physiological recordings were completed, the remaining portions of the D7-S2 spinal cord were removed for microscopic examination to verify that all of the left halves of segments D7-S2 had been completely removed in the earlier surgical procedure. Removal was accomplished by first transecting the whole spinal cord midway along the D6 segment. We held the caudal half of the D6 segment and lifted the cord gently from the canal in a caudal direction. We cut the dorsal and ventral roots as the cord was removed. Midway along the $\mathrm{Ca} 1$ segment, the whole cord was transected to allow complete isolation of the remaining portions of the D7-S2 spinal cord prior to removing the tissue. In five preparations, the right hemicord was transected several additional times and examined with a dissecting microscope. In the fresh tissue, the boundaries of the gray matter and the white matter could be discerned easily; in each of these five preparations, we verified the complete removal of the left half of the spinal cord in segments D7. S2. In two preparations, the right half of the D7-S2 spinal cord was prepared using standard procedures for histological examination of transverse $50 \mu \mathrm{m}$ sections; in both preparations, we verified the com- 
plete removal of the left half of the spinal cord in segments D7-S2. In all seven preparations, we used a camera lucida to sketch each of several sections; in all of these preparations, there was minor damage in the right half of the spinal cord in segments D7-S2. In some locations, there was disruption of axons in the medial part of the right dorsal funiculus and some damage to the medial part of the intermediate right gray matter. The other portions of the right D7-S2 spinal cord displayed a normal morphology.

Analyses for bilateral-recording experiments. Bilateral ENGs obtained in response to electrical stimulation of SP2 were used for detailed analyses; the ENGs were digitized at $2 \mathrm{kHz}$ using Cambridge Electronic Design 1401plus hardware with SPIKE 2 software (Cambridge, UK). The absolute value of the difference between each voltage measurement and the mean voltage for that channel was obtained ("full-wave rectification"); the mean of 20 successive full-wave rectified measurements was calculated ("integrated") so that there were 100 integrated full-wave rectified data points per second. We used custom software written by Dr. Gavin Perry to determine the onset and offset of each burst of integrated full-wave rectified activity. The average amplitude of each burst was obtained by averaging each of the integrated full-wave rectified values occurring within each burst. We analyzed those hip flexor and hip extensor bursts that followed the first full normal cycle of rostral scratching; we only analyzed bursts with onsets and offsets that occurred during stimulation.

We used double-referent phase measurements to calculate the phase of contralateral nerve bursts relative to the activity cycle of the ipsilateral hip flexor. We defined a phase of 0.0 and 1.0 for the events occurring at the onset of the ipsilateral hip flexor burst and 0.5 for events occurring at the offset of the ipsilateral hip flexor burst (Berkowitz and Stein, 1994b). The double-referent phase of an event during an ipsilateral hip flexor burst was the latency of the event from ipsilateral hip flexor onset divided by twice the duration of the ipsilateral hip flexor burst; the double-referent phase of an event during ipsilateral hip flexor quiescence was 0.5 plus the value of the latency of the event from ipsilateral hip flexor offset divided by twice the duration of the ipsilateral hip flexor quiescence. We used vector addition techniques appropriate for circular statistics (Batschelet, 1981; Berkowitz and Stein, 1994b) to obtain the mean vector and the angular deviation (= circular analog of standard deviation) of each set of phase measurements. The angle of the mean vector in radians divided by $2 \pi$ was the mean phase (expressed on a scale from 0.0 to 1.0 ). The length of the mean vector was used in the Rayleigh test to determine the vector's statistical significance (Batschelct, 1981).

We performed two types of phase calculations. In the first type, we calculated average phase for several cycles within several episodes of a specific stimulus condition. We analyzed only full cycles with the normal pattern of rostral scratching that followed the first full normal cycle of rostral scratching; we only analyzed cycles that occurred completely during stimulation. We used Watson's $U^{2}$ test to determine if there was a statistically significant difference between two samples (Batschelet, 1981). In the second type, we calculated the average phase for the first cycle of several episodes of a specific condition, for the second cycle, for the third cycle, and for the fourth cycle. In this type of measurement, we used the side whose hip flexor burst had the earliest onset as the referent. We used the Rank-Sum test for circular data to determine if there was a statistically significant difference between the phase of the first cycle and that of each of the subsequent cycles (Batschelet, 1981).

Analyses for left-hemicord removal experiments. We used a different strategy to analyze the episodes in the left-hemicord removal experiments. Hard copies of stimulus-elicited rostral scratch episodes were printed using a thermal-array recorder (Astro-Med, West Warwick, RI). The number of complete scratch cycles that occurred during stimulation was determined for each episode. Scratch cycles were classified either as normal (rhythmic alternation between hip flexor activity and hip flexor quiescence) or as hip-extensor deletion (no period of hip flexor quiescence between two successive hip flexor bursts). In a normal cycle, a hip flexor (VP-IIP) nerve activity burst preceded a period of hip flexor quiescence. In a hip-extensor deletion cycle, the burst of hip flexor activity was followed immediately by the burst of hip flexor activity of the next cycle; there was not a quiescent period between two sequential bursts and the hip extensor (HR-KF) burst normally occurring at this time was absent. In these cases, the first burst of hip flexor activity was counted as belonging to a hip-extensor deletion cycle if (1) there was an obvious low-amplitude region of the hip flexor ENG and (2) there

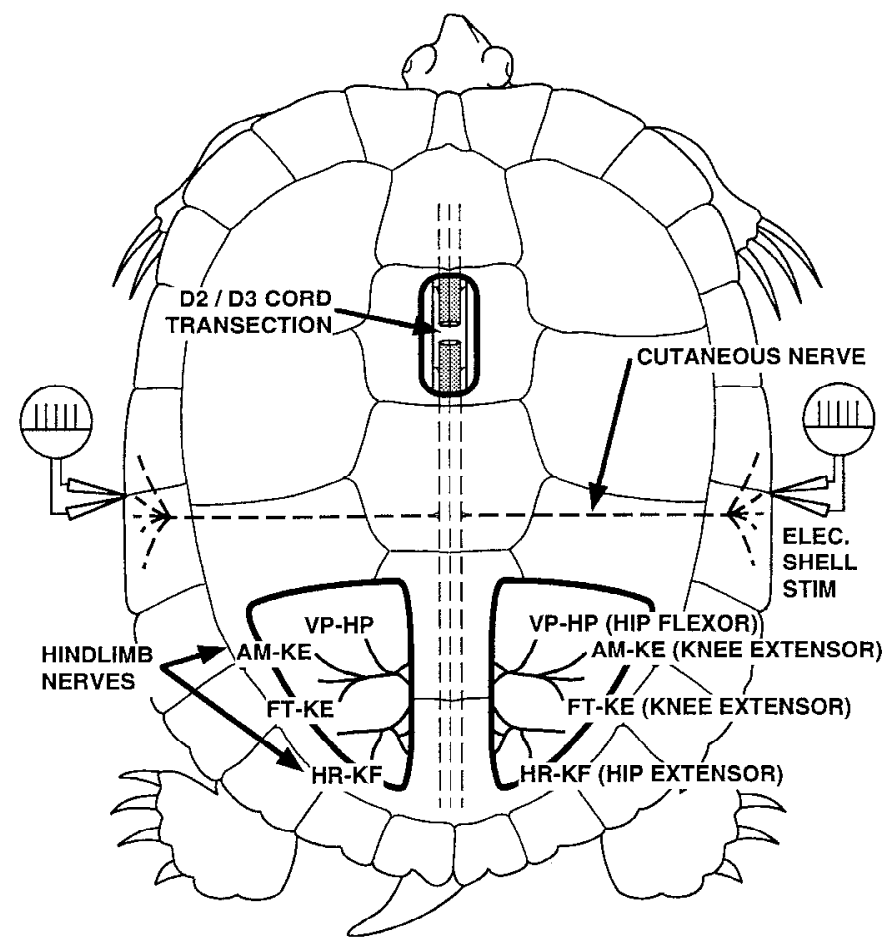

Figure 1. Illustration of the bilateral-recording preparation. The spinal cord was completely transected just posterior to the forelimb enlargement. Bilateral recordings were obtained from hip flexor $(V P-H P)$, hip extensor $(H R-K F)$, and knee extensor $(A M-K E$ and/or $F T-K E)$ nerves. The sketch illustrates the electrical pulses that were used in some episodes to stimulate in the left and/or the right rostral scratch receptive fields. In other episodes, mechanical stimulation was used

was a separate knee extensor (AM-KE or HT-KE) burst associated with each high-amplitude region of the hip flexor ENG. Only cycles that occurred completely within the period of stimulation were included in the analysis. For normal cycles, the offset of the hip extensor burst or the onset of the next hip flexor burst needed to occur during stimulation; for hip-extensor deletion cycles, the onset of the next hip flexor burst needed to occur during stimulation. Using this classification technique, we calculated the percentage of the total number of cycles in each episode that expressed the hip-extensor deletion. For each turtle, we calculated the average of these percentages for episodes evoked by electrical stimulation as well as a separate average of these percentages for episodes evoked by mechanical stimulation. For each condition in each turtle, we tested the statistical significance of the difference between the control percentages and the left D7-S2 removed percentages with the one-tailed Mann-Whitney $U$ test (Siegel, 1956).

\section{Results}

\section{Bilateral motor output during unilateral stimulation and} during bilateral stimulation

Bilateral ENGs of motor output during fictive rostral scratching were obtained in 11 turtles using the preparation schematically presented in Figure 1. In all 11 turtles, we recorded hip flexor (VP-HP) and knee extensor (FT-KE or AM-KE) ENGs bilaterally; in 9 of 11 turtles, we also recorded hip extensor (HR-KF) ENGs bilaterally. Unilateral cutaneous stimulation of a site in the rostral scratch receptive field elicited a vigorous pattern of fictive rostral scratching in motor nerves ipsilateral to the site of stimulation (Fig. 2A,C; see also Robertson et al., 1985). The response of right hip nerves to stimulation in the right receptive field shown in Figure $2 C$ was the "normal" pattern of rostral scratching that included rhythmic alternation between hip flexor and hip extensor activity. In the normal rostral scratch, activation 


\section{A LEFT STIM}

LEFT KNEE EXTENSOR

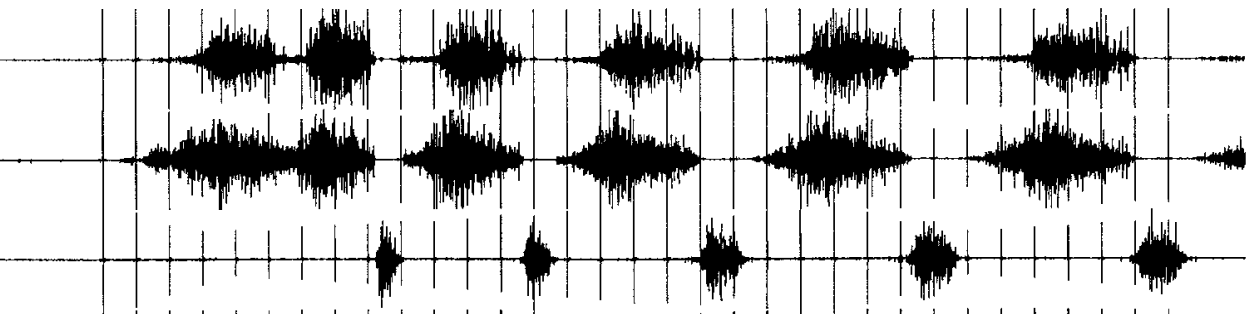
RIGHT KNEE EXTENSOR

RIGHT HIP FLEXOR

RIGHT HIP EXTENSOR
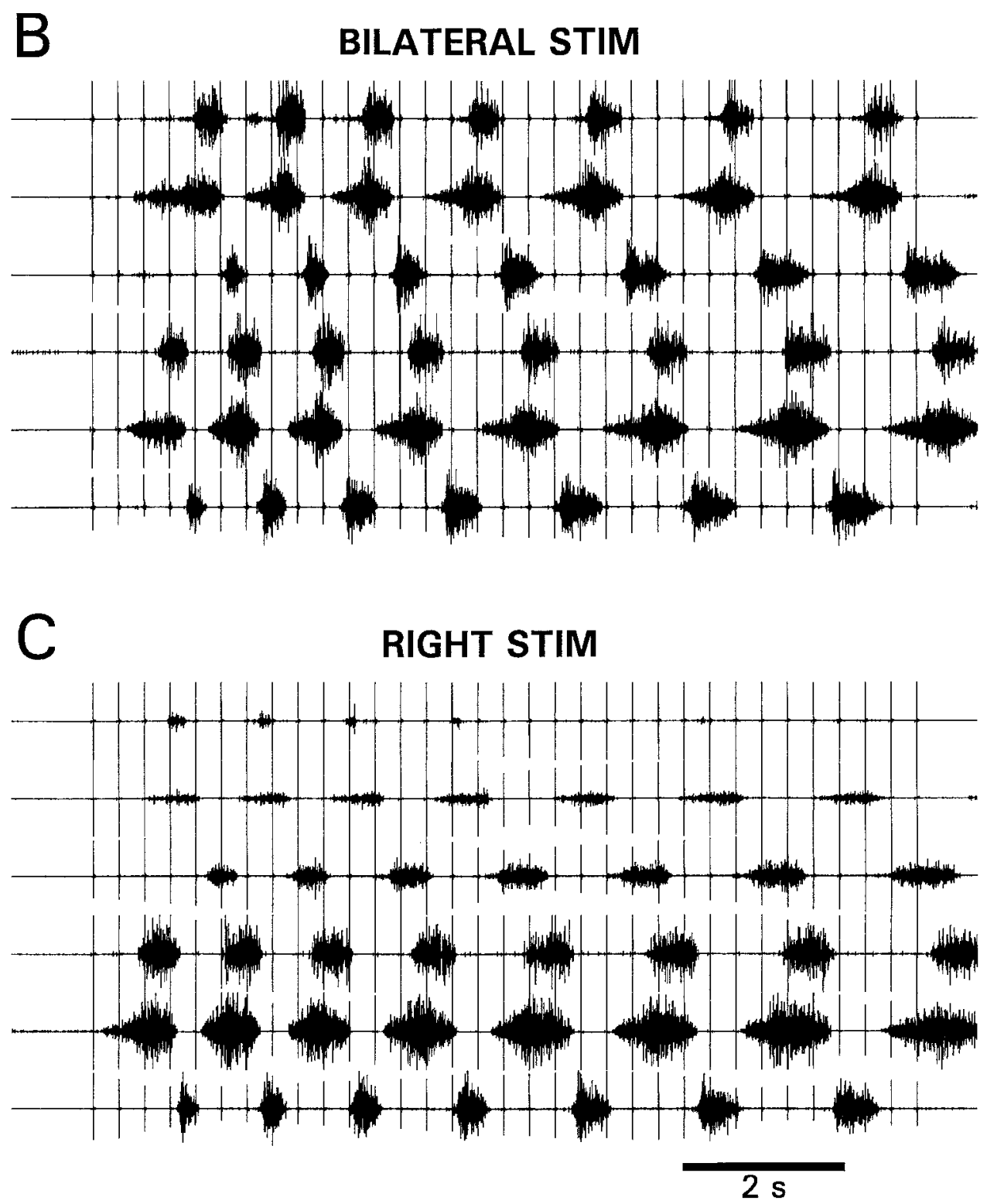
of the ipsilateral knee extensor occurred during the latter portion of the ipsilateral hip flexor burst. The response of left hip nerves to stimulation in the left receptive field shown in Figure $2 A$ began with the "hip-extensor deletion" motor pattern characterized by two successive bursts of hip flexor activity without an intervening period of hip flexor quiescence (see also Fig. 2 of Robertson and Stein, 1988). Subsequent cycles of the episode in Figure $2 A$ displayed the normal pattern of rostral scratching. In both examples of unilateral stimulation, the first nerve to be activated was the hip flexor nerve ipsilateral to the site of the stimulation (Fig. $2 A, C$ ).

For each example of unilateral stimulation, there was modest activation of hip nerves contralateral to the site of stimulation (for descriptions of contralateral hip flexor activities see also Currie and Stein, 1989; Berkowitz and Stein, 1994a). Activation of the contralateral hip extensor alternated with activation of the contralatcral hip flexor (Fig. $2 A, C$ ); this was observed in nine of nine turtles. Activation of the contralateral hip extensor occurred during the initial portion of ipsilateral hip flexor activation (nine of nine turtles). Activation of the latter portion of the contralateral hip flexor burst occurred during ipsilateral hip extensor activation ( 9 of 11 turtles); in 2 of 11 turtles, no contralateral hip flexor activity was observed during unilateral stimulation. Occasionally in some turtles, the contralateral knee extensor (top trace in Fig. $2 C$ ) was also activated with low amplitude during contralateral hip flexor activity.

Bilateral cutaneous stimulation of a site in the left rostral scratch receptive field and of a mirror-image site in the right rostral scratch receptive field elicited a vigorous pattern of rostral scratching in both the left motor nerves and the right motor nerves (Fig. $2 B$ ). Initially, there was simultaneous activation of both the left hip flexor nerve and the right hip flexor nerve. After the first cycle on each side, activation of a given nerve alternated with activation of the corresponding contralateral nerve. We observed this pattern of coordinated activity in 11 of 11 turtles. Similar patterns of coordination are also observed during bilateral rostral scratching movements (Field and Stein, 1994). This out-of-phase motor pattern during bilateral rostral scratching resembles the out-of-phase coordination of hindlimbs during stepping and swimming movements observed in intact turtles (Walker, 1971; Zug, 1971; Davenport et al., 1984; Field and Stein, 1994)

Measurements of the amplitude and phase of the motor patterns. We analyzed bilateral ENGs during fictive rostral scratching in response to electrical stimulation in three turtles. In these turtles, we calculated average values of amplitude and phase for both hip flexor and hip extensor ENGs. We measured the average amplitude of the rectified ENG during each burst of motor activity. The average amplitude of a given nerve in response to an ipsilateral stimulus was taken as the $100 \%$ value for each turtle. See Materials and Methods for a complete description of the procedure. For all measurements of hip flexor (HF) amplitude in the three turtles (Fig. 3A), the average amplitude of a given hip flexor nerve for a contralateral stimulus was considerably less (range of average values, $11-31 \%$ ) than the amplitude of the same nerve for an ipsilateral stimulus. These differ- ences were statistically significant at the $p<0.001$ level for each of the three turtles. In all three turtles, the average amplitude of a given hip extensor (HE) nerve for a contralateral stimulus was less than the amplitude of the same nerve for an ipsilateral stimulus (Fig. 3B). For two of three turtles, these differences were significant; range of average values in these turtles was $25-75 \%$. For unilateral stimulation, the responses of hip motor nerves to a contralateral stimulus were generally of lower amplitude than those to an ipsilateral stimulus; thus, the bilateral motor output in response to a unilateral rostral scratch stimulus was asymmetric.

We also compared the average amplitude of a given nerve in response to a unilateral stimulus to the average amplitude of the same nerve in response to bilateral stimuli. For hip flexor recordings in all three turtles, the average amplitudes for bilateral stimuli were similar to that for a unilateral stimulus (Fig. 3A). For hip extensor recordings in all three turtles, the average amplitudes for bilateral stimuli were greater than the amplitude of the same nerve for an ipsilateral stimulus (Fig. 3B). For two of three turtles, these differences were significant; range of average values in these turtles was $127 \%$ to $158 \%$. Thus, simultaneous ipsilateral and contralateral stimuli produced a higher intensity of hip extensor activity than either stimulus alone.

We used double-referent phase analysis to describe the timing of contralateral motor output within the ipsilateral hip flexor activity cycle (Fig. 4). Double referent phase analysis is appropriate to situations with a variable duty cycle (= duration of hip flexor burst divided by cycle period). See Materials and Methods for a description of phase measurement procedures; Berkowitz and Stein (1994b) also provide detailed justification for doublereferent analyses for turtle fictive scratching. In these analyses, the onset of the ipsilateral hip flexor burst was defined as 0.0 and 1.0 phase and the offset of the same burst was defined as 0.5 phase. An event in the middle of the hip flexor burst had a value of 0.25 ; an event in the middle of hip flexor quiescence had a value of 0.75 . These measurements revealed that, during unilateral stimulation, contralateral hip extensor activity began near the onset of ipsilateral hip flexor activity and ended during the latter portion of the ipsilateral hip flexor burst (Fig. 4). Contralateral hip flexor activity began during the latter portion of the ipsilateral hip flexor burst and ended near the onset of the next ipsilateral hip flexor burst. Thus, contralateral hip activity was mainly out-of-phase with the corresponding ipsilateral hip motor activity. There was a portion of each cycle in which there was an overlap of left and right hip flexor activities, however; during the latter portion of the ipsilateral hip flexor burst, there was also activation of the contralateral hip flexor nerve. There will be overlapping portions of an out-of-phase pattern if both left and right duty cycles are greater than 50\%. Another example of overlap during out-of-phase coordination is the double-support phase of human walking; double support occurs when the beginning of one leg's stance phase overlaps with the end of the other leg's stance phase (Herman et al., 1975).

When the phase of contralateral motor activity during bilateral stimulation was compared with the corresponding phase during unilateral stimulation, there was a modest phase shift in the onset

Figure 2. Bilateral recordings of fictive rostral scratching ENGs in response to electrical pulses delivered to the SP2 positions in the rostral scratch receptive fields on the left side $(A)$, on both sides $(B)$, and on the right side $(C)$. In each example, ENGs on both the left and the right sides for knee extensor $(F T-K E)$, hip flexor $(V P-H P)$, and hip extensor $(H R-K F)$ nerves are shown. At each site of stimulation, 33 pulses with 320 msec interpulse interval, $10 \mathrm{~V}$ amplitude, and $1 \mathrm{msec}$ duration were delivered. Stimulus artifacts mark the timing of each stimulus pulse. 

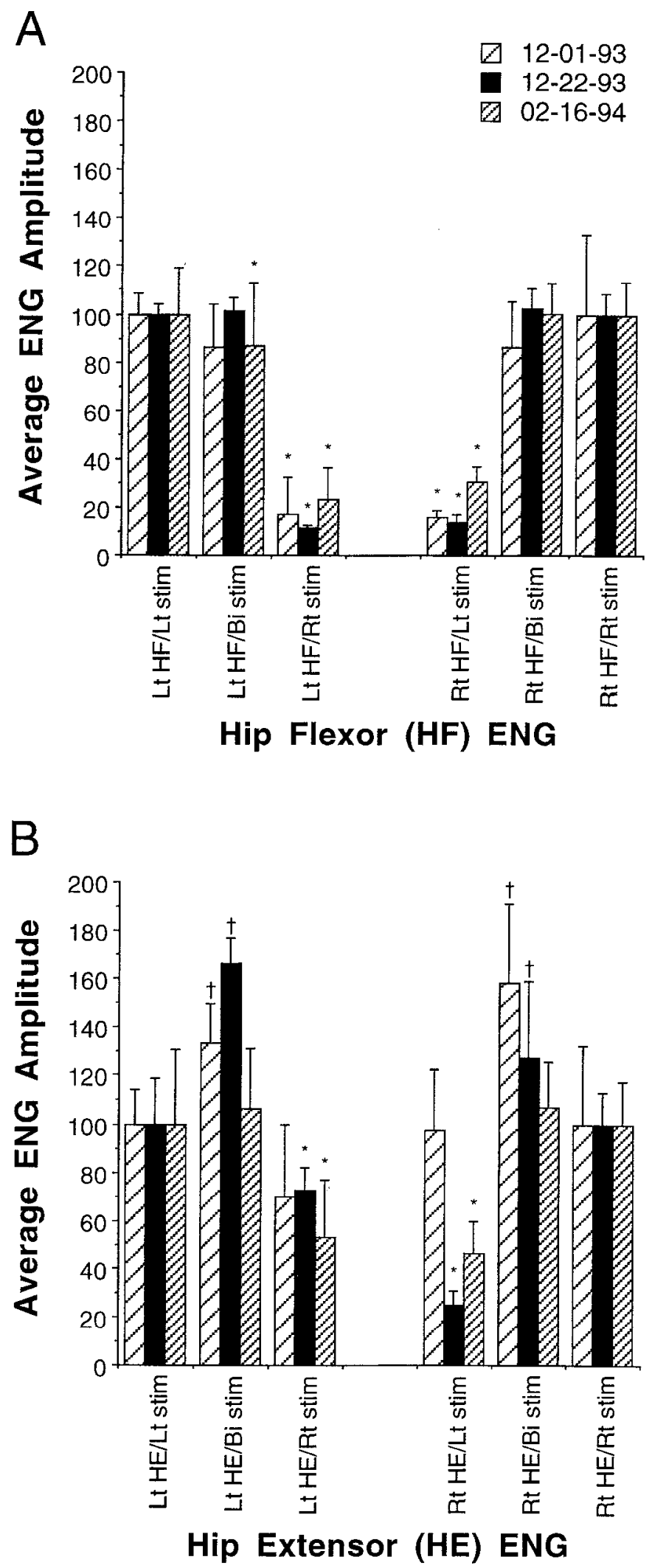

Figure 3. Graphs of average burst amplitude of hip flexor and hip extensor nerves during fictive rostral scratching for left unilateral ( $L t$ stim), bilateral (Bi stim), and right unilateral (Rt stim) stimulation conditions. Each bar represents amplitude of activity for each nerve under each condition; data from three separate experiments are presented. A, Graph of average hip flexor nerve hurst amplitude for the three stimulation conditions. Left hip flexor ( $L t H F$ ) average burst amplitude values and right hip flexor (Rt HF) average burst amplitude values are nor- of contralateral hip extensor activity and a corresponding phase shift in the offset of the contralateral hip flexor activity (Fig. 4). For both of these cases, there was a shift from a phase value near 0.0 to a phase value in the early portion of the hip flexor burst. In all three turtles, these shifts were statistically significant.

The measurements displayed in Figure 4 are phase measurements from cycles that followed the first complete normal cycle (see Materials and Methods for criteria for inclusion of cycles in these phase measurements). During each episode of bilateral stimulation, the phase of the initial cycle was different from the phase of subsequent cycles. In Figure 5, the onset phase of the contralateral hip flexor burst was plotted as a function of the cycle number in the episode. We analyzed data from four turtles for these analyses. For the first cycle of the episode, the onset phase was slightly greater than 0.0 ; for subsequent cycles of the episode, the onset phase was near $0.3-0.4$. Thus, the initial cycle of each episode displayed in-phase coordination of left and right hip flexors; the subsequent cycles of each episode displayed outof-phase coordination of these nerves. Stein (1978) examined forelimb/hindlimb coordination during swimming movements elicited by electrical stimulation of the spinal cord in a highspinal turtle; this preparation displayed a shift from an in-phase initial response to out-of-phase responses in subscquent cycles. Similar shifts are also observed in analyses of ankle movements during human stepping when in-phase ankle movements prior to lift-off are compared with out-of-phase ankle movements during rhythmic stepping (Herman et al., 1973).

Right hindlimb motor output in response to unilateral stimulation in the absence of the left half of the spinal cord hindlimb enlargement

Recordings of right hindlimb motor output during fictive rostral scratching after removal of the left halves of six spinal segments were obtained in seven turtles using the preparation illustrated in Figure $6 A$. We removed the left halves of the five segments of the hindlimb enlargement (D8, D9, D10, S1, S2), as well as the left half of the D7 segment just anterior to the hindlimb enlargement. A camera lucida sketch of a representative crosssection through the remaining right half of the D9 segment is presented in Figure $6 B$.

We designed this preparation so that we could deliver either an ipsilateral stimulus in the right rostral scratch receptive field or a contralateral stimulus in the left rostral scratch receptive field (Fig. 6A). Primary afferents that travel in the left and in the right dorsal roots of spinal segments D3-D6 innervate the left and the right rostral scratch receptive fields, respectively (Mortin and Stein, 1990). Some propriospinal interneurons with axons that descend in the white matter of the right hindlimb enlargement have somata in left gray matter of segments D3-

malized to their unilateral ipsilateral stimulation condition, $L t$ stim and Rt stim, respectively. Vertical error bars indicate the values of the standard deviation for the average amplitude. $B$, Graph of average hip extensor ( $L t H E$ and $R t H E$ ) nerve burst amplitude for the three stimulation conditions. Data are normalized to the respective unilateral ipsilateral stimulation conditions as in A. Asterisks indicate that the average amplitude is less than the unilateral ipsilateral stimulation condition at a level of $p<0.001$ by the one-tailed Mann-Whitney $U$ test for linear data (Siegel, 1956). Daggers indicate that the average amplitude is greater than the unilateral ipsilateral stimulation condition, at a level of $p<0.001$ by the one-tailed Mann-Whitney $U$ test for linear data. 
A

(a) 12-01-93

$12-22-93$

( $02-16-94$

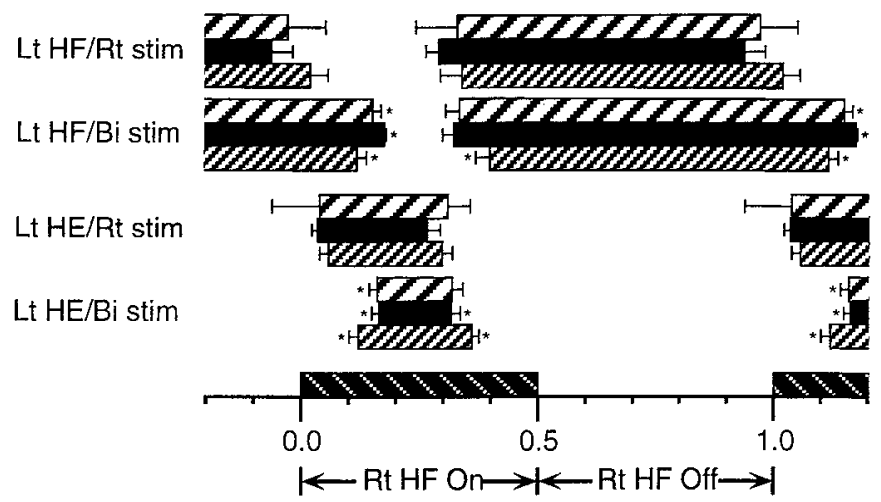

B

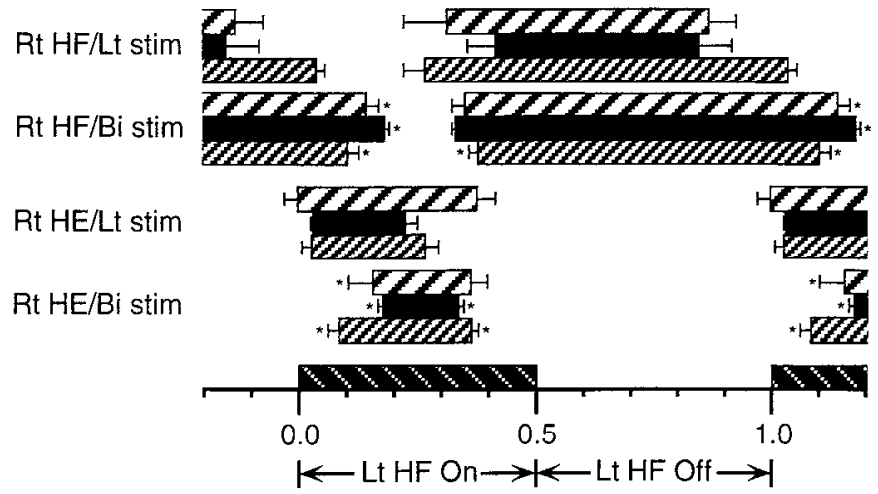

Figure 4. Graphs of average onset and offset phases of contralateral hip flexor and contralateral hip extensor bursts during the normal pattern of fictive rostral scratching with respect to two referents of the ipsilateral hip flexor cycle for either unilateral or bilateral electrical shell stimulation. A, Left hip flexor and extensor data relative to the right hip flexor cycle. The top set of data represents left hip flexor data $(L t H F)$ for right unilateral electrical shell stimulation (Rt stim); the next set of data represents Lt HF for bilateral electrical shell stimulation ( $\mathrm{Bi}$ stim). The next two sets of tata represent left hip extensor data $(L t H E)$ for the same stimulation conditions, respectively. Shaded rectangles represent bursts of activity for each nerve under each condition; three separate experiments are presented. The left edge of each rectangle represents the average burst onset, while the right edge of each rectangle represents the average burst offset, relative to the right hip flexor (Rt HF $O n / O f f)$ cycle. $B$, Right hip flexor $(R t H F)$ and extensor $(R t H E)$ data relative to the left hip flexor cycle ( $L t H F O n / O f f)$. Data is organized similarly to the data in $A$. For both $A$ and $B$. The data used to calculate each onset and offset value presented in this graph were significantly different from a random distribution $(p<0.001$, Rayleigh test; Batschelet, 1981). Horizontal error bars indicate the values of the average angular deviation for nerve burst onset or offset. Asterisks indicate that average onset or offset values for bilateral stimulation are significantly different than for unilateral stimulation at a level of $p<0.001$ by the Watson $U^{2}$ test for circular data (Batschelet, 1981).

D6; others have somata in the right gray matter of these segments (Berkowitz and Stein, 1994c). Thus, in the preparation with the left halves of segments D7-S2 removed, the D3-D6 segments that innervate both left and right rostral scratch receptive fields remain intact and in communication with the right halves of segments D7-S2. The next section describes responses of this preparation to stimulation in the right rostral scratch re-

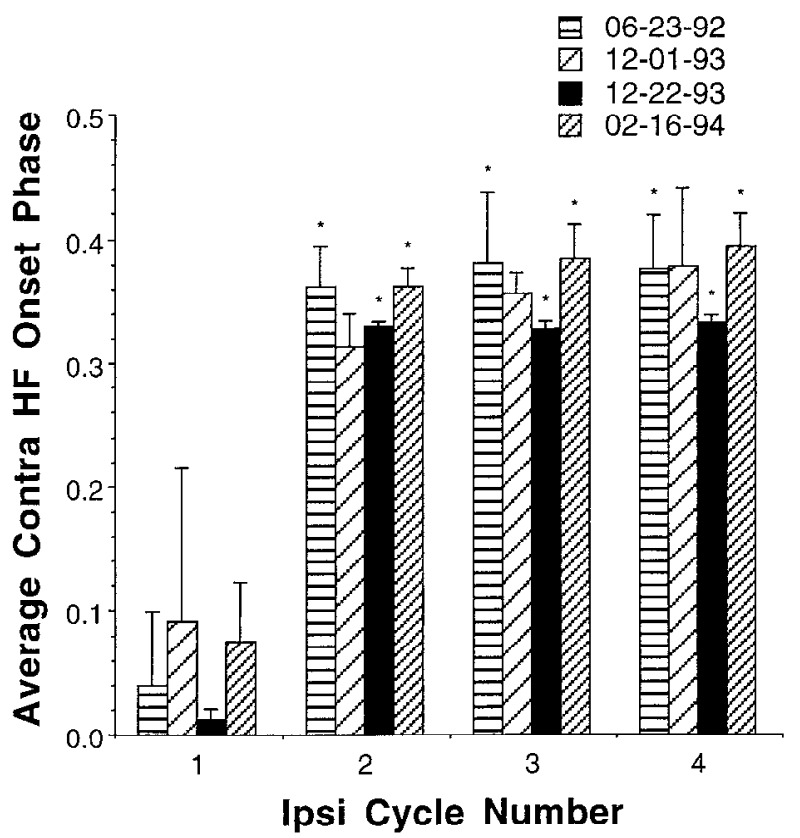

Figure 5. Graph of average contralateral hip flexor onset phase relative to the ipsilateral hip flexor cycle for four consecutive cycles of bilateral fictive rostral scratching in response to bilateral electrical shell stimulation. Bars represent the average phase of contralateral hip flexor burst onset (Average Contra IIF Onset Phase) in double-referent mode. Data from four experiments are illustrated. The side whose hip flexor burst occurred with shortest latency following the beginning of bilateral stimulation was defined as the ipsilateral referent. The data used to calculate each value presented in this graph were significantly different from a random distribution $(p<0.001$, Rayleigh test; Batschelet, 1981). Vertical error bars indicate the values of average angular deviation for burst onset. Asterisks indicate that the values of average hip flexor burst onset in the second, third, and fourth cycles are significantly different than the value in the first cycle at a level of $p<0.001$ by the RankSum test for circular data (Batschelet, 1981).

ceptive field; the subsequent section describes responses to stimulation in the left rostral scratch receptive field.

Responses to ipsilateral stimulation. Prior to left-hemicord reInoval, control recordings from right hindlimb nerves were obtained in response to cutaneous stimulation in the right rostral scratch receptive field. In the episode displayed in Figure $7 A$, all cycles of the response displayed the normal ipsilateral pattern of rostral scratching consisting of rhythmic alternation between hip flexor activation and hip flexor quiescence (see also the bottom three traces in Fig. 2C). In these cycles, hip extensor activation occurred during hip flexor quiescence; knee extensor activation occurred in the latter portion of each hip flexor burst. The column labeled "Control" in Table 1 summarizes the average percentage of cycles per episode that displayed hip-extensor deletions. These recordings displayed a very low percentage of cycles with hip-extensor deletions, that is, they were characterized mainly by the normal pattern of rostral scratching consisting of rhythmic alternation between hip flexor activation and hip flexor quiescence.

Subsequent to left-hemicord removal, recordings from right hindlimb nerves were obtained in response to stimulation in the right rostral scratch receptive field. Rhythmic bursts of hip flexor activity were observed after left-hemicord removal; activation of knee extensor activity occurred during the latter portion of each hip flexor burst (Fig. $7 B$ ). The hip extensors were not active; there was no hip flexor quiescence between hip flexor bursts. 


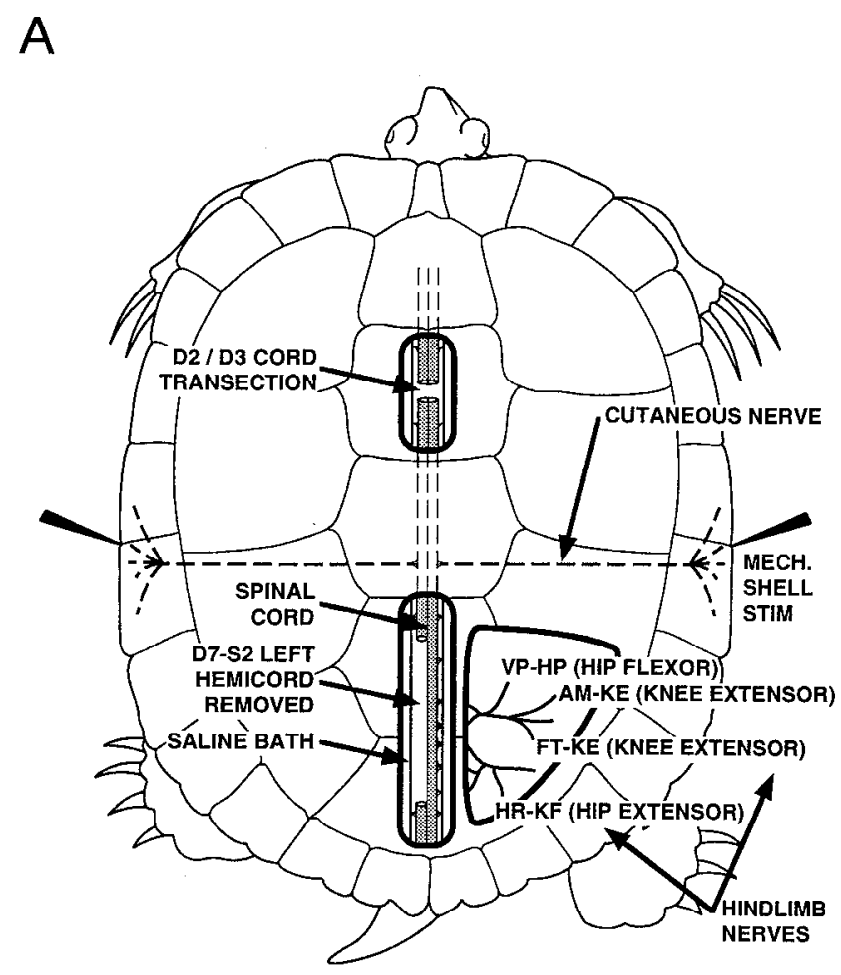

B

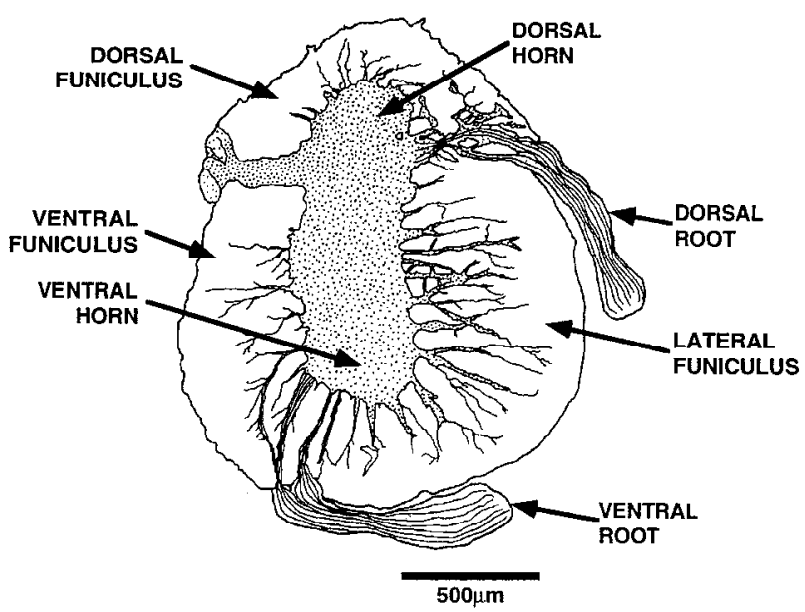

Figure 6. Illustration of the preparation and lesion used for left-hemicord removal experiments. A, Illustration of the preparation with complete spinal transection posterior to the forelimb enlargement. The segments of the hindlimb enlargement $(D 8-S 2)$ were exposed as was the D7 segment. ENGs of the right knee extensor ( $A M-K E$ and/or $F T-K E)$, hip flexor $(V P-H P)$, hip extensor $(H R-K F)$ muscle nerves were recorded during either ipsilateral or contralateral shell stimulation. Shown is mechanical shell stimulation used in some episodes; in other episodes, electrical shell stimulation was used. The sketch shows the preparation after the left D7-S2 hemicord has been removed; the preparation was also uscd prior to left-hemicord removal for control recordings. $B$, Camera lucida drawing of a representative cross-section of the right D9 hemicord in a preparation with left-hemicord removal.

This motor pattern, the hip-extensor deletion variation of fictive rostral scratching, was produced for all cycles of this response. Thus, the left half of the hindlimb enlargement was not necessary for the production of rhythmic hip flexor bursts that occur during the hip-extensor deletion variation of fictive rostral scratching. This rcsult is consistent with the hypothesis that neural circuitry, located in the right half of the spinal cord and termed the right hip flexor module, can produce the rhythm of right hip flexor activity in response to stimulation in the right rostral scratch receptive field.

In the example illustrated in Figure $7 B$, all cycles of the response displayed the hip-extensor deletion variation of rhythmic ficlive rostral scratching. This variation of fictive rostral scratching was observed in all of the left-hemicord removed preparations tested. The column labeled "Left D7-S2 Removed" in Table 1 describes the average percentage of cycles per episode that displayed hip-extensor deletions after left-hemicord removal; in this condition, there was a very high percentage of hip-extensor deletions. Very few cycles of the normal motor pattern of fictive rostral scratching were observed after left-hemicord removal; thus, the left half of the spinal cord was required for the routine production of the normal pattern of fictive rostral scratching. These results therefore support the idea that neuronal circuitry in the left half of the spinal cord contributed to the production of the normal pattern of fictive rostral scratching in right hindlimb nerves. The CPG for right hindlimb rostral scratching is the set of spinal cord neurons that contribute to the production of the normal pattern of fictive right hindlimb rostral scratching. These results are thus consistent with the hypothesis that there is a bilateral set of neurons in the spinal cord that contribute to the right rostral scratching CPG, that is, neurons in the left half of the spinal cord as well as neurons in the right half of the spinal cord may be members of the right rostral scratching CPG.

Responses to contralateral stimulation. Prior to left-hemicord removal, control recordings from right hindlimb nerves were obtained in response to cutaneous stimulation of the left rostral scratch receptive field. In the episode displayed in Figure $8 A$, all cycles of the response displayed a normal pattern of contra lateral hindlimb motor activity in response to stimulation of a site in the ipsilateral rostral scratch receptive field (see also the bottom three traces in Fig. $2 A$ ). Strong rhythmic activation of hip extensor activity alternated with lower-amplitude hip flexor activity.

Subsequent to left hemicord removal, recordings from right hindlimb nerves were obtained in response to stimulation of the left rostral scratch receptive field. Rhythmic bursts of right hip extensor activity were still observed even after left-hemicord removal (Fig. 8B). Thus, the left half of the hindlimb enlargement was not necessary for the production of right hip extensor rhythmic activity in response to left rostral scratch receptive field stimulation. This result was observed in three of seven preparations. In these three preparations, weak rhythmic hip flexor activity was also observed. In one of the other preparations, weak rhythmic hip flexor activity was observed even though there was no hip extensor activity. In the remaining three preparations, no rhythmic activity was observed. Our observations of rhythmic hip extensor activity in three of seven preparations support the hypothesis that neural circuitry, located in the right half of the spinal cord and termed the right hip extensor module, can produce the rhythm of right hip extensor activity in response to stimulation in the left rostral scratch receptive field.

\section{Discussion}

Contralateral hip nerves were rhythmically activated during stimulation in the ipsilateral rostral scratch receptive field; the contralateral motor rhythm was out-of-phase with the ipsilateral fictive rostral scratching motor rhythm (Fig. $2 A, C$ ). Activation 
Table 1. Average percentage of cycles with hip extensor deletions per stimulation episode

\begin{tabular}{|c|c|c|c|c|c|c|}
\hline \multirow{3}{*}{$\frac{\text { Experiment }}{62493(\mathrm{E})}$} & \multicolumn{3}{|l|}{ Control } & \multicolumn{3}{|c|}{ Left D7-S2 removed } \\
\hline & \multirow{2}{*}{$\begin{array}{l}\text { No. of } \\
\text { episodes }\end{array}$} & \multicolumn{2}{|c|}{$\begin{array}{l}\text { Ave. percentage of } \\
\text { cycles per episode } \\
\text { with hip extensor } \\
\text { deletions (SD) }\end{array}$} & \multirow{2}{*}{$\begin{array}{l}\text { No. of } \\
\text { episodes }\end{array}$} & \multicolumn{2}{|c|}{$\begin{array}{l}\text { Ave. percentage of } \\
\text { cycles per episode } \\
\text { with hip extensor } \\
\text { deletions (SD) }\end{array}$} \\
\hline & & 4.2 & (11.8) & & 97.9 & $(8.3)$ \\
\hline $7-1-93(\mathrm{E})$ & 6 & 0 & $(0)$ & 12 & 100 & $(0)$ \\
\hline $7-22-93(E)$ & 7 & 0 & $(0)$ & 6 & 100 & $(0)$ \\
\hline $8-11-93(E)$ & 7 & 0 & $(0)$ & 6 & 100 & $(0)$ \\
\hline $9-20-93(\mathrm{E})$ & 8 & 0 & (0) & 16 & 100 & $(0)$ \\
\hline $10-26-93(\mathrm{E})$ & 8 & 0 & (0) & 9 & 100 & (0) \\
\hline $11-9-93(E)$ & 8 & 0 & (0) & 15 & 100 & $(0)$ \\
\hline $6-24-93(\mathrm{M})$ & 3 & 0 & $(0)$ & 19 & 46.6 & (15.2) \\
\hline $7-1-93(\mathrm{M})$ & 6 & 0 & $(0)$ & 12 & 100 & $(0)$ \\
\hline $7-22-93(\mathrm{M})$ & 7 & 0 & (0) & 6 & 94.4 & $(13.6)$ \\
\hline $8-11-93(\mathrm{M})$ & 7 & 0 & $(0)$ & 9 & 100 & (0) \\
\hline $9-20-93(\mathrm{M})$ & 8 & 0 & (0) & 16 & 97.7 & (6.3) \\
\hline $10-26-93(\mathrm{M})$ & 8 & 5 & $(9.3)$ & 8 & 100 & $(0)$ \\
\hline $11-9-93(\mathrm{M})$ & 8 & 0 & (0) & 16 & 100 & (0) \\
\hline
\end{tabular}

This table compares control cycles with an intact hindlimb enlargement versus cycles after removal of the left D7S2 spinal segments. For each preparation, we used either electrical (E) or mechanical (M) stimulation in the right rostral scratch receptive field to elicit fictive rostral scratching in right hindlimb nerves. Average percentages and their SDs were calculated from percentages of cycles per episode showing hip extensor deletions (see Materials and Methods). For each stimulus condition in each preparation, the significance of "Left D7-S2 removed" values was tested against "Control" values using the one-tailed Mann-Whitney $U$ test (Siegel, 1956). In all cases, $p<0.001$.

of contralateral motor neurons was most likely produced, in part, by contralateral excitatory interneurons. It is a reasonable hypothesis that some of these contralateral interneurons may be members of the ipsilateral limb's rostral scratch CPG; this hypothesis received further support from our results following the removal of six left hemisegments (Figs. $7 B, 8 B$ ).

The result that, in response to stimulation in the right rostral scratch receptive field, the hip-extensor phase of the normal pattern of right hindlimb rostral scratching was missing after lefthemicord removal (Fig. $7 B$ ) supports the concept that an important part of the right rostral scratch CPG is located in the left hemicord. The result that left segments D7-S2 are not needed for the thythm of right hip extensors in response to stimulation in the left rostral scratch receptive field (Fig. $8 \mathrm{~B}$ ) indicates that the ipsilateral limb's rostral scratch CPG may include a contralateral neural circuit that generates a rhythm of contralateral hip extensor bursts. In addition, the result that rhythmic right hip flexor activation in response to stimulation in the right rostral scratch receptive field was produced after left-hemicord removal (Fig. $7 B$ ) supports the concept that ipsilateral circuitry of an ipsilateral limb's rostral scratch CPG may include a neural circuit that generates a rhythm of ipsilateral hip flexor bursts. Taken together, these observations provide experimental support for the concept that an ipsilateral limb's rostral scratch CPG includes an ipsilateral hip flexor rhythm generator that is coupled to a contralateral hip extensor thythm generator. The present work adds to previous studies in spinal motor physiology (see Sherrington 1906, 1910) that emphasize the linkage of ipsilateral flexion with contralateral extension.

The present article therefore supports the concept that interneurons that are members of the rostral scratch CPG for one hindlimb are distributed bilaterally. A mechanism that can generate such a distribution is that some spinal neurons on one side of the spinal cord may be members of both the right rostral scratch CPG and the left rostral scratch CPG. A spinal neuron that is "shared" by both rostral scratch CPGs would be rhythmically active in response to stimulation in either the right or the left rostral scratch receptive field. Many descending axons of turtle propriospinal interneurons were rhythmically active in response to stimulation in either an ipsilateral or a contralateral scratch receptive field; others were activated by stimulation of only one side (Berkowitz and Stein, 1994a,b). The simplest hypothesis consistent with both these unit-recording data and the present article's data is that there is partial overlap between the left rostral scratch $C P G$ and the right rostral scratch $C P G$, that is, some members of one CPG are also members of the other CPG.

We introduce the term "bilateral shared core" to describe rostral scratch CPG neurons that are members of both rostral scratch CPGs. A schematic of the hip interneurons that may be members of this bilateral shared core, as well as the motor neurons activated by these interneurons, is presented in Figure 9. According to this point of vicw, (1) when the right rostral scratch receptive field is stimulated, the CPG neurons of the bilateral shared core are activated along with the unshared CPG neurons of the right rostral scratch $\mathrm{CPG}$; and (2) when the left rostral scratch receptive field is stimulated, the CPG neurons of the bilateral shared core are activated along with the unshared $\mathrm{CPG}$ neurons of the left rostral scratch CPG. The "bilateral shared core" model presented in Figure 9 is only a partial representation of the rostral scratch CPGs; unshared CPG neurons are not illustrated in Figure 9. A more complete model of the rostral scratch CPG is required to account for the timing of other motor pools, for example, knee extensors (see Robertson et al., 1985; Berkowitz and Stein, 1994b). The rostral scratch bilateral shared core may also be involved in the generation of other motor rhythms, for example, the pocket scratch (Berkowitz and Stein, 1994a,b; Field and Stein, 1994). 


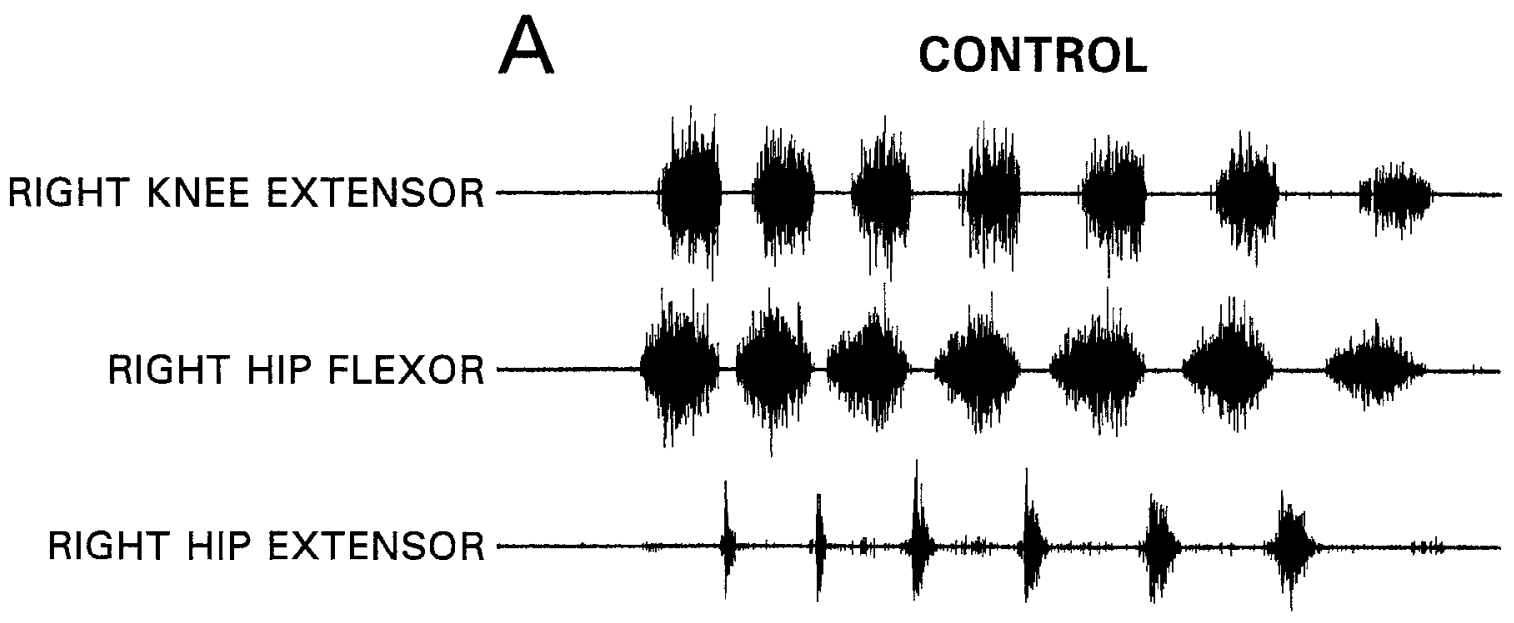

RIGHT STIM
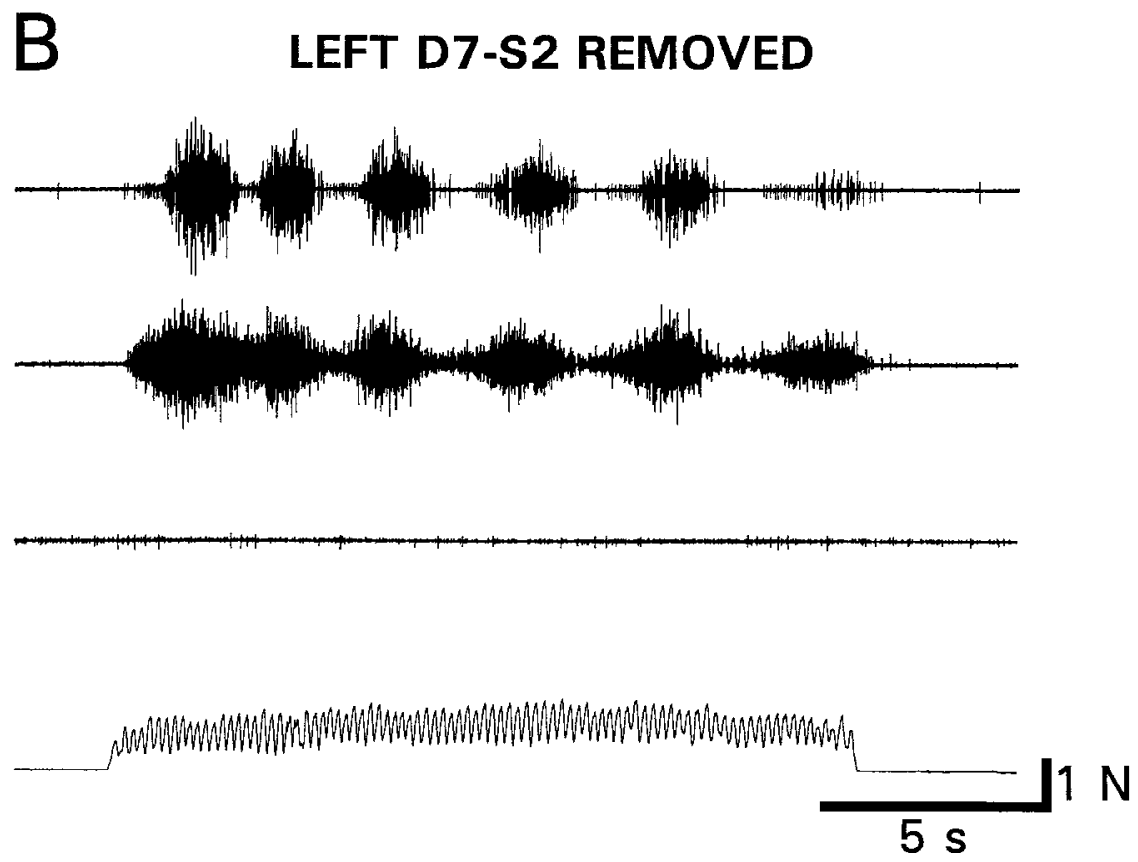

Figure 7. Ipsilateral recordings in response to ipsilateral stimulation in a contralateral-hemicord removal preparation. ENGs from right knee extensor $(A M-K E)$, hip flexor $(V P-H P)$, and hip extensor $(H R-K F)$ nerves in response to mechanical stimulation of a site in the right rostral scratch receptive field. $A$, Control recordings prior to left-hemicord removal. $B$, Recordings after left-hemicord removal of segments D7-S2.

The type of organization presented in Figure 9 has been termed a "modular" organization (Jordan et al., 1986; Jordan, 1991; also termed "unit burst generator" by Grillner, 1981) since excitatory interneurons and inhibitory interneurons that are co-active with a motor pool form a control module for that motor pool. The concept of a module was introduced to explain the relationship between the excitatory drive to a motor pool and the inhibitory drive to its antagonist motor pool. In particular, excitatory interneurons of the module may excite each other as well as agonist motor neurons; some excitatory interneurons of the module may excite inhibitory interneurons that inhibit antagonist motor neurons. Intracellular recordings from hip motor neurons during the hip-extensor deletion variation of fictive ros- tral scratching provide direct evidence for such modular organization (Stein et al., 1982; Robertson and Stein, 1988). Strong support for this modular organization as a general feature of vertebrate CPG organization comes from direct interneuronal recordings from spinal neurons during swimming (Roberts et al., 1986; Grillner et al., 1991).

The concept of a bilateral ncuronal core shared by left and right limb CPGs departs from traditional ideas concerning neural control of interlimb coordination (Stein, 1976; Grillner, 1981). Traditional views assume no sharing of neural elements between the CPG for one limb and the CPG for another limb; interlimb phase control is a result of coordinating neuron output from one CPG that synaptically modulates neural elements in the other 


\section{A CONTROL}
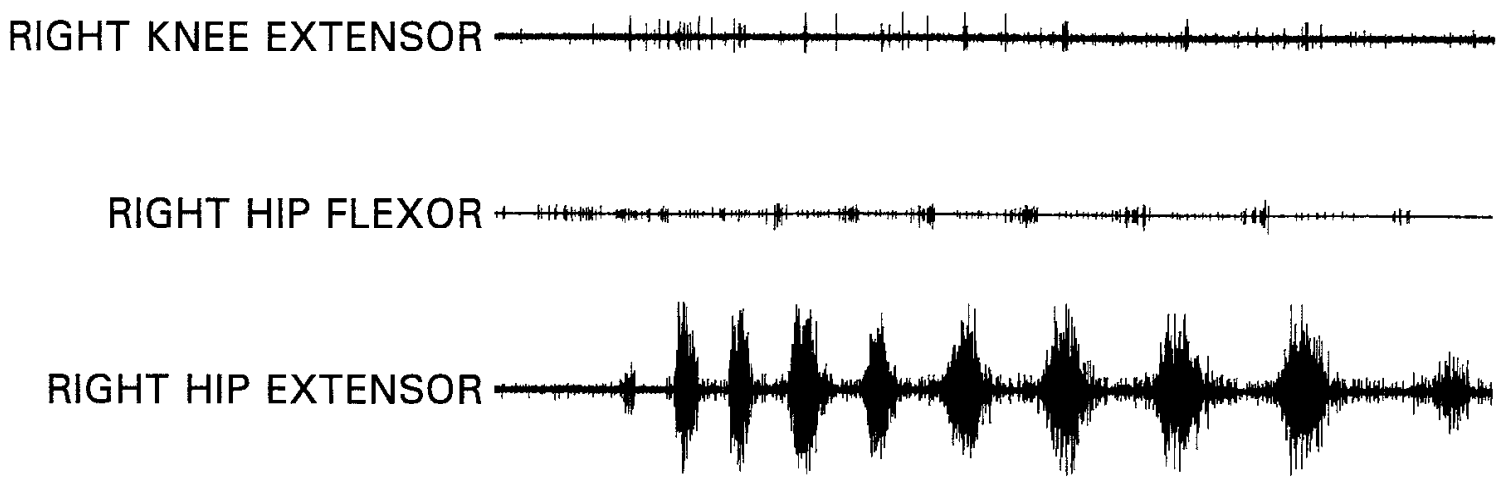

LEFT STIM

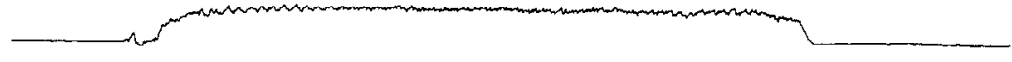

B LEFT D7-S2 REMOVED
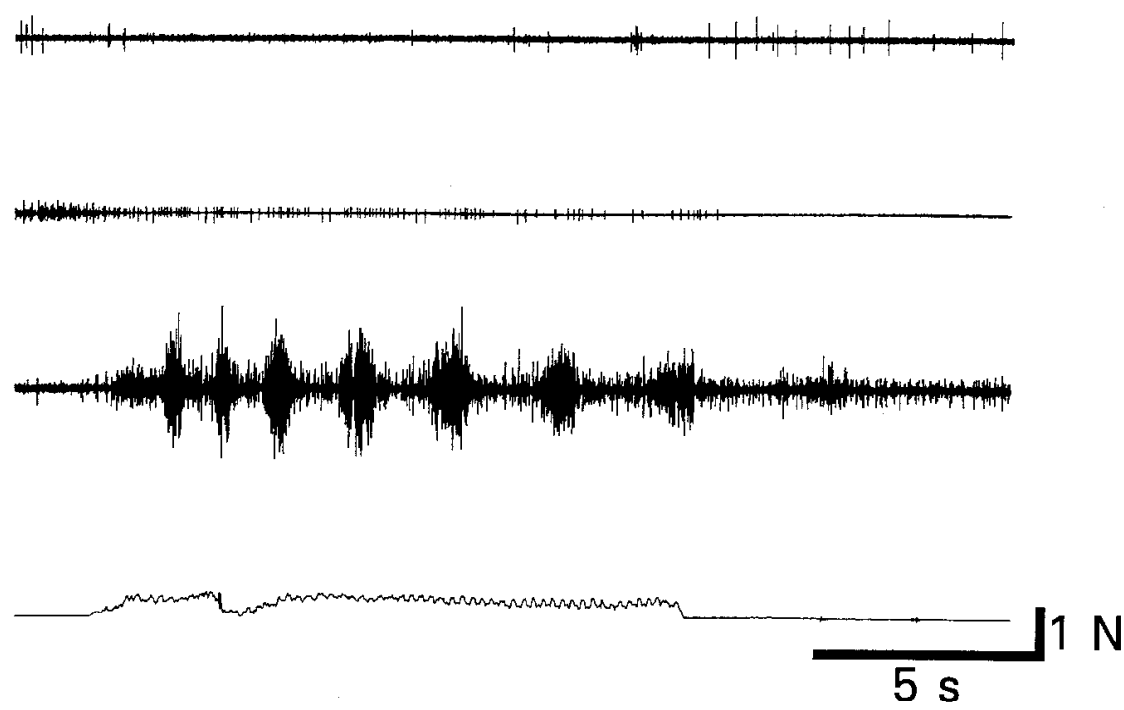

Figure 8. Ipsilateral recordings in response to contralateral stimulation in a contralateral-hemicord removal preparation. ENGs from right knee extensor $(A M-K E)$, hip flexor $(V P-H P)$, and hip extensor $(H R-K F)$ nerves in response to mechanical stimulation of a site in the left rostral scratch receptive field. $A$, Control recordings prior to left-hemicord removal, $B$, Recordings after left-hemicord removal of segments D7-S2.

CPG. According to the view in the present article, neural elements that may coordinate interlimb phase are embedded into the structure of each limb's CPG via the bilateral shared core. When both CPGs are fully activated, connections among the shared core may be sufficient to ensure an appropriate interlimb phase. This article demonstrated a proper out-of-phase pattern of interlimb coordination in response to bilateral rostral scratch receptive field stimulation (Fig. $2 B$ ). This model system may assist future experimental tests of new theoretical models of interlimb phase control.

How does the rhythm generator in the contralateral hindlimb enlargement interact with the rhythm generator in the ipsilateral hindlimb enlargement to produce a normal ipsilateral rostral scratch motor pattern? Insight into a possible mechanism may be obtained by examining bilateral recordings during fictive rostral scratching produced by an intact hindlimb enlargement. These recordings demonstrate that during rostral scratching evoked by stimulation in the right rostral scratch receptive field there is left hip flexor activation during right hip flexor quiescence (Figs. 2C, 4). Several mechanisms of left/right interaction may contribute to this interlimb phase relationship. A mechanism relevant to the present article is that inhibitory left enlargement interneurons, that are coactive with left hip flexor activation, may inhibit right enlargement neurons that are coactive with right hip flexor activation (Fig. 9); this inhibition may assist in the production of right hip flexor quiescence. Thus, the ab- 


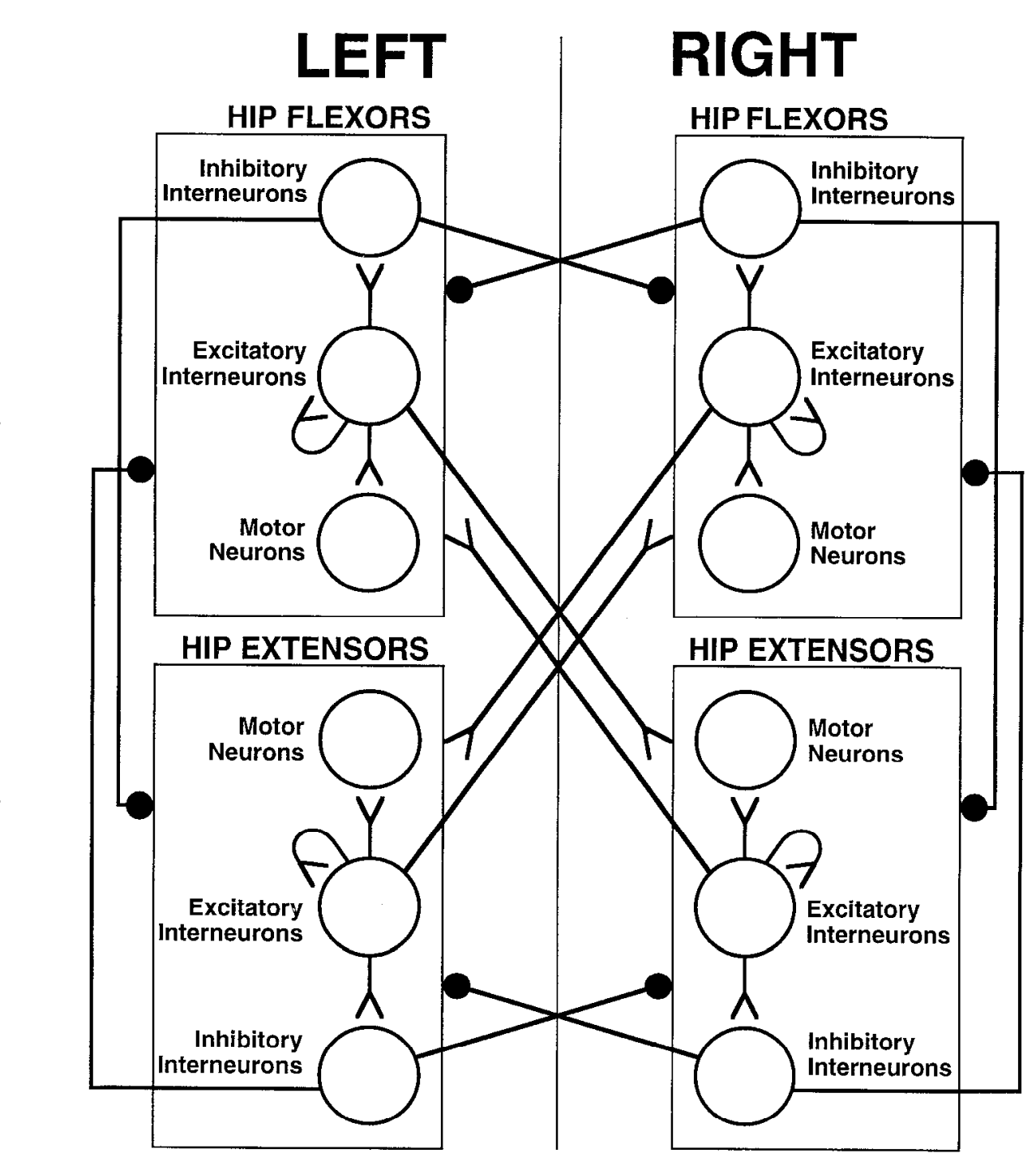

Figure 9. Sketch of the proposed organization of the hip modules of the rostral scratch bilateral shared core in the turtle spinal cord hindlimb enlargement. Illustrated as members of the bilateral shared core are CPG hip interneurons that are activated when either the left or the right rostral scratch receptive field is stimulated. Shown also are hip motor neurons that are activated under either stimulus condition. Neurons are represented with modular organization; members of each module are enclosed in a rectangle. Members of a given module are activated synergically via the outputs of some of the module's excitatory interneurons; excitation is represented as a fork. In addition, others of the module's excitatory interneurons may also excite memopposite type of movement. Some of each module's inhibitory interneurons inhibit members of the ipsilateral antagonistic module; others of each module's inhibitory interneurons inhibit members of the contralateral module for the same type of movement; inhibition is represented as a solid circle. Neurons in this bilateral core for the rostral scratch CPGs may also be shared with other bilateral CPGs, perhaps those of other scratch forms and/ or those of each of several forms of locomotion. bers of the contralateral module for the

sence of right hip flexor quiescence observed in the present paper when the left enlargement is removed may be due, in part, to the removal of left enlargement inhibitory interneurons that normally contribute to right hip flexor quiescence. The importance of contralateral sources of inhibition for ipsilateral axial motor control is well established (Roberts et al., 1986; Fetcho, 1991; Grillner et al., 1991). Our results suggest evolutionary persistence of similar influences for hip motor control (Cohen, 1988).

Mortin and Stein (1989) also observed the hip-extensor deletion variation of fictive rostral scratching after bilatcral rcmoval of all spinal segments posterior to segment D8. Their result was consistent with the hypothesis that neural circuitry responsible for activation of hip extensors was located in segment D9 and in more posterior segments. When that circuitry was removed, they reasoned there may have been a loss of an inhibitory signal coupled to hip extensor activation that normally contributcs to the hip flexor quiescence observed in the normal pattern of fictive rostral scratching. Thus, the results of Mortin and Stein (1989) and the present results taken together support the idea that at least two sources of inhibition onto neurons active during ipsilateral hip flexor motor output are required during rostral scratching for the production of rhythmic alternation between ipsilateral hip flexor activation and ipsilateral hip flexor quiescence: an ipsilateral source linked to ipsilateral hip extension and a contralateral source linked to contralateral hip flexion. Both sources are indicated in Figure 9.

We also propose mutual excitation between the ipsilateral hip extensor module and the contralateral hip flexor module (Fig. 9). During the production of the normal pattern of ipsilateral rostral scratching with an intact hindlimb enlargement, this mutual excitation may contribute directly to the excitability of the ipsilateral hip extensor module and the contralateral hip flexor module; increased excitability of these modules may, in turn, contribute to the production of ipsilateral hip flexor quiescence.

The right half of the turtle hindlimb enlargement contains a circuit that can generate the hip-extensor variation of fictive rostral scratching that is characterized by rhythmic hip flexor bursts. The rhythmogenic capacity of the ipsilateral part of the nervous system has been demonstrated in invertebrates (Ronacher, 1989; Murchison et al., 1993) and in vertebrates (Kudo and Yamada, 1987; Soffe, 1989; Kudo el al., 1991). Thus, the ipsilateral nervous system contains a neural circuit that generates rhythmic motor output in an ipsilateral limb in many organisms. The current study also presents evidence that supports the hypothesis that contralateral circuitry makes a significant contribution to the production of the normal pattern of ipsilateral rostral scratching in the turtle. It will be important to examine, in detail, the 
motor patterns in other organisms to determine if contributions from contralateral circuitry also play a significant role in the production of the normal motor pattern of the ipsilateral limb.

\section{References}

Arshavsky YI, Gelfand IM, Orlovsky GN, Pavlova GA (1978) Messages conveyed by spinocerebellar pathways during scratching in the cat. II. Activity of neurons of the ventral spinocerebellar tract. Brain Res 151:493-506.

Barajon I, Gossard JP, Hultborn H (1992) Induction of fos expression by activity in the spinal rhythm generator for scratching. Brain Res 588:168-172.

Batschelet E (1981) Circular statistics in biology. New York: Academic.

Berkowitz A, Stein PSG (1994a) Activity of descending propriospinal axons in the turtle hindlimb enlargement during two forms of fictive scratching: broad tuning to regions of the body surface. J Neurosci 14:5089-5104.

Berkowitz A, Stein PSG (1994b) Activity of descending propriospinal axons in the turtle hindlimb enlargement during two forms of fictive scratching: phase analyses. J Neurosci 14:5105-5119.

Berkowitz A, Stein PSG (1994c) Descending propriospinal axons in the hindlimb enlargement of the red-eared turtle: cells of origin and funicular courses. J Comp Neurol 346:321-336.

Cohen AH (1988) Evolution of the vertebrate central pattern generator for locomotion. In: Neural control of thythmic movements in vertebrates (Cohen AH, Rossignol S, Grillner S, eds), pp 129-166. New York: Wiley.

Currie SN, Stein PSG (1989) Interruptions of fictive scratch motor rhythms by activation of cutaneous flexion reflex afferents in the turtle. J Neurosci 9:488-496.

Currie SN, Stein PSG (1990) Cutaneous stimulation evokes long-lasting excitation of spinal interneurons in the turtle. J Neurophysiol 64: 1134-1148.

Davenport J, Munks SA, Oxford PJ (1984) A comparison of swimming in marine and freshwater turtles. Proc R Soc Lond [Biol] 220:447475 .

Deliagina TG, Orlovsky GN, Perret C (1981) Efferent activity during fictitious scratch reflex in the cal. J Neurophysiol 45:595-604.

Fetcho JR (1991) Spinal network of the Mauthner cell. Brain Behav Evol 37:298-316.

Field EC, Stein PSG (1994) Spinal cord control of bilateral hindlimb coordination in the turtle. Soc Neurosci Abstr 20:1754.

Grillner S (1981) Control of locomotion in bipeds, tetrapods, and fish. In: Handbook of physiology, Sect 1, The nervous system, Vol 2, Motor control (Brooks VB, ed), pp 1179-1236. Bethesda, MD: American Physiological Society.

Grillner S, Wallen P, Brodin L, Lansner A (1991) Neuronal network generating locomotor behavior in lamprey: circuitry, transmitters, membrane properties, and simulation. Annu Rev Neurosci 14:169199.

Herman RM, Cook T, Cozzens B, Freedman W (1973) Control of postural reactions in man: the initiation of gait. In: Control of posture and locomotion (Stein RB, Pearson KG, Smith RS, Redford JB, eds), pp 363-388. New York: Plenum.

Herman RM, Wirta R, Bampton S, Finley FR (1975) Human solutions for locomotion: single limb analysis. In: Neural control of locomotion (Herman RM, Grillner S, Stein PSG, Stuart DG, eds), pp 13-49. New York: Plenum.

Jordan LM (1991) Brainstem and spinal cord mechanisms for the initiation of locomotion. In: Neurobiolngical hasis of human locomotion (Shimamura M, Grillner S, Edgerton VR, eds), pp 3-20. Tokyo: Japan Scientific Societies.

Jordan LM, Brownstone RM, Kriellaars DJ, Noga BR (1986) Spinal modules for walking movements revealed in fictive locomotion experiments. Soc Neurosci Abstr 12:877.

Kudo N, Yamada T (1987) N-Methyl-D,L-aspartate-induced loconotor activity in a spinal cord-hindlimb muscles preparation of the newborn rat studied in vitro. Neurosci Lett 75:43-48.

Kudo N, Ozaki S, Yamada T (1991) Ontogeny of rhythmic activity in the spinal cord of the rat. In: Neurobiological basis of human locomotion (Shimamura M, Grillner S, Edgerton VR, eds), pp 127-136. Tokyo: Japan Scientific Societies.

Melby ECJ, Altman NH (1974) Handbook of laboratory animal science, Vol 1. Cleveland: CRC.

Mortin LI, Stein PSG (1989) Spinal cord segments containing key elements of the central pattern generators for three forms of scratch reflex in the turtle. J Neurosci 9:2285-2296.

Mortin LI, Stein PSG (1990) Cutaneous dermatomes for the initiation of three forms of the scratch reflex in the spinal turtle. J Comp Neurol 295:515-529.

Mortin I,I, Keifer J, Stein PSG (1985) Three forms of the scratch reflex in the spinal turtle: movement analyses. J Neurophysiol 53:15011516.

Murchison D, Chrachri A, Mulloney B (1993) A separate local patterngenerating circuit controls the movements of each swimmeret in crayfish. J Neurophysiol 70:2620-2631.

O’Donovan MJ, Pinter MJ, Dum RP, Burke RE (1982) Actions of FDL and FHL muscles in intact cats: functional dissociation between anatomical synergists. J Neurophysiol 47:1126-1143.

Roberts A, Soffe SR, Dale N (1986) Spinal interneurones and swimming in frog embryos. In: Neurobiology of vertebrate locomotion (Grillner S, Stein PSG, Stuart DG, Forssberg H, Herman RM, eds), pp 279-306. London: Macmillan.

Robertson GA, Stein PSG (1988) Synaptic control of hindlimb motoneurones during three forms of the fictive scratch reflex in the turtle. J Physiol (Lond) 404:101-128.

Robertson GA, Mortin LI, Keifer J, Stein PSG (1985) Three forms of the scratch reflex in the spinal turtle: central gencration of motor patterns. J Neurophysiol 53:1517-1534.

Ronacher B (1989) Stridulation of acridid grasshoppers after hemisection of thoracic ganglia: evidence for hemiganglionic oscillators. $J$ Comp Physiol 164A:723-736.

Ruigrok TJH, Crowe A (1984) The organization of motoneurons in the turtle lumbar spinal cord. J Comp Ncurol 228:24-37.

Sherrington CS (1906) The integrative action of the nervous system. New Haven: Yale UP.

Sherrington CS (1910) Flexion-reflex of the limb, crossed extension reflex, and reflex stepping and standing. J Physiol (Lond) 40:28-121.

Siegel S (1956) Nonparametric statistics for the behavioral sciences. New York: McGraw-Hill.

Soffe SR (1989) Roles of glycinergic inhibition and $N$-methyl-D-aspartate receptor mediated excitation in the locomotor rhythmicity of one-half of the Xenopus embryo central nervous system. Eur $J$ Neurosci 1:561-571.

Stcin PSG (1976) Mcchanisms of interlimb phase control. In: Neural control of locomotion (Herman RM, Grillner S, Stein PSG, Stuart DG, eds), pp 465-487. New York: Plenum.

Stein PSG (1978) Swimming movements elicited by electrical stimulation of the turtle spinal cord: the high spinal preparation. J Comp Physiol 124:203-210.

Stein PSG (1989) Spinal cord circuits for motor pattern selection in the turtle. Ann NY Acad Sci 563:1-10.

Stein PSG, Grossman ML (1980) Central program for scratch reflex in turtle. J Comp Physiol 140:287-294.

Stein PSG, Robertson GA, Keifer J, Grossman ML, Berenbeim JA, Lennard PR (1982) Motor neuron synaptic potentials during fictive scratch reflex in turtle. J Comp Physiol 146:401-409.

Stein PSG, Victor JC, Field EC, Currie SN (1994) Bilateral motor rhythms during fictive rostral scratching in the turtle. Soc Neurosci Abstr 20:1754.

Walker WF (1971) A structural and functional analysis of walking in the turtlc, Chrysemys picta marginata. J Morphol 134:195-214.

Zug GR (1971) Buoyancy, locomotion, morphology of the pelvic girdle and hindlimb, and systematics of cryptodiran turtles. Misc Publ Mus Zool Univ Mich 142:1-98. 